\title{
On the functional form of particle number size distributions: influence of particle source and meteorological variables
}

\section{Katia Cugerone et al.}

Correspondence to: Katia Cugerone (katia.cugerone@ polimi.it)

The copyright of individual parts of the supplement might differ from the CC BY 3.0 License. 


\section{Supplement A}

\section{Domain of a mixture of two lognormals in the skewness-kurtosis plane}

Let assume that $X$ follows a mixture of two lognormal distributions. Its density $f_{X}(x)$ is

$$
f_{X}(x)=\sum_{i=1}^{2} \pi_{i} f_{i}(x)
$$

where $f_{i}(x)$ and $\pi_{i}$, are respectively the (lognormal) density and the weight of the $i$-th component. The (positive) weights must satisfy the condition $\sum_{i=1}^{2} \pi_{i}=1$. The $k$-th order moment respect the origin of the mixture $E\left[X^{k}\right]=\mu^{(k)}$ has been calculated as

$$
\mu^{(k)}=\sum_{i=1}^{2} \pi_{i} \mu_{i}^{(k)}
$$

where $\mu_{i}^{(k)}$ is the $k$-th order moment respect the origin of the $i$-th component. $\mu_{i}^{(2)}, \mu_{i}^{(3)}, \mu_{i}^{(4)}$ can be written in terms of mean $\mu_{i}^{(1)}$, standard deviation $\sigma_{i}$, skewness $\beta_{3_{i}}$ and kurtosis $\beta_{4_{i}}$ of the $i$-th component as

$$
\begin{gathered}
\mu_{i}^{(2)}=\sigma_{i}^{2}+\left(\mu_{i}^{(1)}\right)^{2} \\
\mu_{i}^{(3)}=\beta_{3_{i}} \sigma_{i}^{3}+3 \mu_{i}^{(1)} \sigma_{i}^{2}+\left(\mu_{i}^{(1)}\right)^{3} \\
\mu_{i}^{(4)}=\beta_{4_{i}} \sigma_{i}^{4}+4 \mu_{i}^{(1)} \beta_{3_{i}} \sigma_{i}^{3}+6\left(\mu_{i}^{(1)}\right)^{2} \sigma_{i}^{2}+\left(\mu_{i}^{(1)}\right)^{4} .
\end{gathered}
$$


The skewness $\beta_{3}$ and kurtosis $\beta_{4}$ of the mixture are respectively

$$
\begin{gathered}
\beta_{3}=\frac{\mu^{(3)}-3 \mu^{(1)} \mu^{(2)}+2\left(\mu^{(1)}\right)^{3}}{\left(\mu^{(2)}-\left(\mu^{(1)}\right)^{2}\right)^{3 / 2}} \\
\beta_{4}=\frac{\mu^{(4)}-4 \mu^{(1)} \mu^{(3)}+6 \mu^{(2)}\left(\mu^{(1)}\right)^{2}-3\left(\mu^{(1)}\right)^{4}}{\left(\mu^{(2)}-\left(\mu^{(1)}\right)^{2}\right)^{2}} .
\end{gathered}
$$

Substituting Eq.s (A.3-A.5) into Eq.s (A.6-A.7), it is possible to express skewness and kurtosis of the mixture as a function of mean, standard deviation, skewness and kurtosis of each component. The domain of a mixture of two lognormal distributions in $\beta_{3}-\beta_{4}$ plane has been determined numerically through a Montecarlo simulation by simulating couples of lognormal distributions with parameter $\mu$ in the range (-10:0.1:10), parameter $\sigma$ in the range (0:0.1:10) and weight $\pi$ in the range (0:0.1:10). The skewness and the kurtosis can be calculated by using equations $(4,5)$.

The resulting theoretical domain of a mixture of two lognormals distribution is an area, embracing the simple lognormal domain. 


\section{Supplement B}

\section{Skewness-kurtosis plane figures}

This figure shows the location of the sample couples $\left(\beta_{3}, \beta_{4}\right)$ from MI1 (a), MI2 (b), MI4 (c), MI5 (d), MI6 (e) and MI7 (f) datasets in the skewnesskurtosis plane with theoretical distribution reference domains. Cyan dots represent minutes with $\mathrm{TP}<25000$, blue dots $25000<\mathrm{TP}<62500$, purple dots $62500<\mathrm{TP}<100000$ and magenta dots $\mathrm{TP}>100000$. 

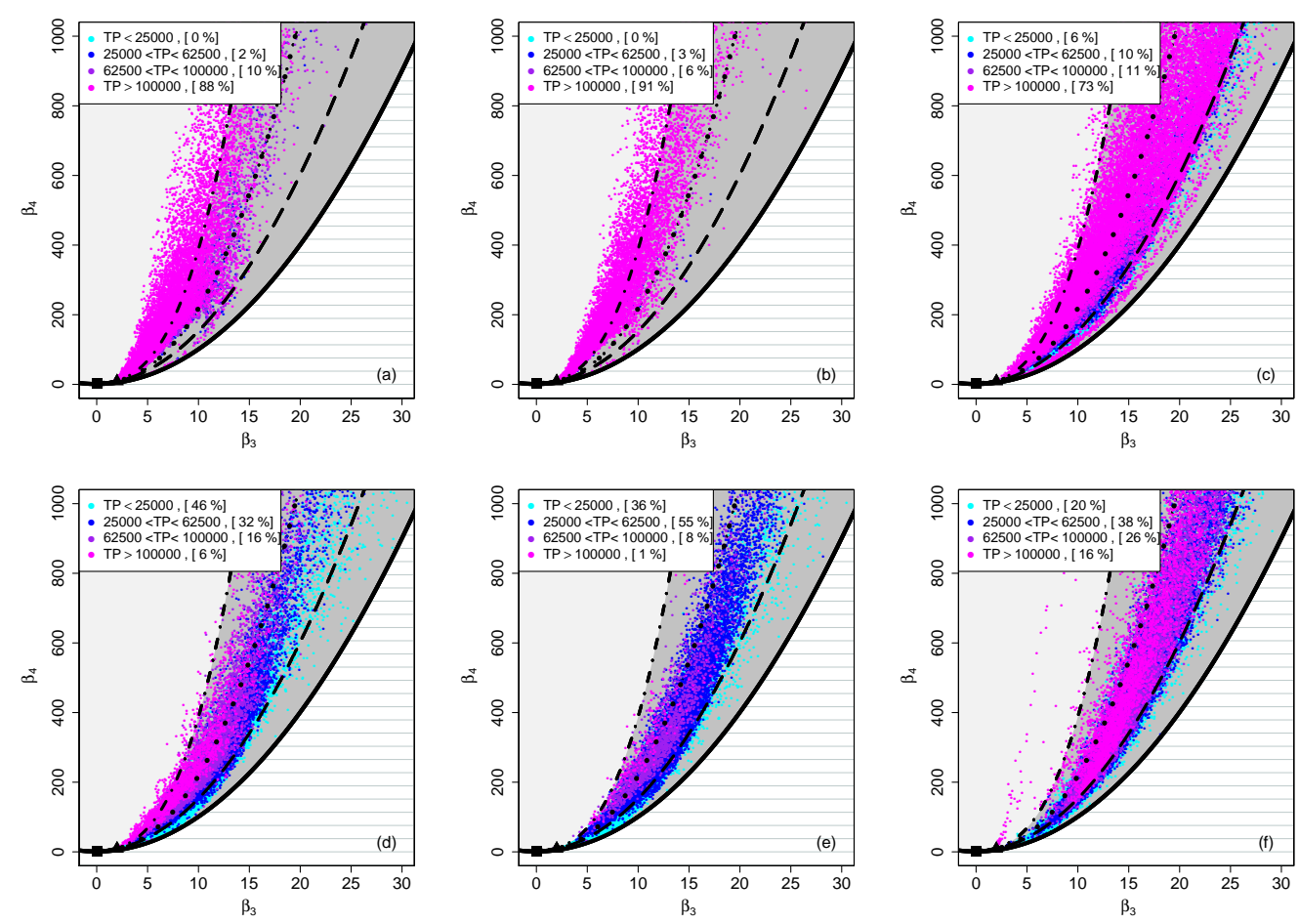

Figure B.1: Location of the sample couples $\left(\beta_{3}, \beta_{4}\right)$ datasets in the skewnesskurtosis plane with theoretical distribution reference domains. The dotteddashed line is the lognormal distribution, the dotted line is the Weibull distribution, and the dashed line is the gamma distribution. 


\section{Supplement C}

\section{JSB distribution characteristics and fitting method}

The Johnson SB distribution is part of the Johnson's system of distributions, made known by Johnson [4], as a flexible model, able to cover a wide variety of distribution shapes. This system is composed by three families, SB (System Bounded), SU (System Unbounded) and SL (System Lognormal), so that any dataset with finite moments can be fitted by one of them. The probability density function of a Johnson $\mathrm{SB}$ variate $X$ is:

$$
p(x)=\frac{\delta}{\sqrt{2 \pi}} \cdot \frac{\lambda}{(x-\xi)(\lambda+\xi-x)} \cdot \exp \left\{-\frac{1}{2}\left[\gamma+\delta \ln \left(\frac{x-\xi}{\lambda+\xi-x}\right)\right]^{2}\right\}
$$

with $x \in[\xi, \xi+\lambda],-\infty<\xi<\infty, \lambda>0,-\infty<\gamma<\infty$ and $\delta>0$. $\xi$ and $\lambda$ are respectively the location and scale parameters, $\xi$ and $(\xi+\lambda)$ can be considered proportional to the minimum and maximum value of the variable. $\gamma$ and $\delta$ affect the shape of the distribution.

We use the Maximum Likelihood (ML) method to estimate the parameters of the Johnson SB. Starting from the density function of the Johnson SB, Eq.C.1, the log-likelihood function can be written as:

$$
\begin{aligned}
& L^{*}=N \ln (\lambda)+N \ln (\delta)+N \ln (2 \pi)^{-1 / 2}-N \gamma^{2} / 2-\sum \ln \left(x_{i}-\xi\right)- \\
& \sum \ln \left(\xi+\lambda-x_{i}\right)-\gamma \delta \sum \ln \left(x_{i}-\xi\right)+\gamma \delta \sum \ln \left(\xi+\lambda-x_{i}\right) \\
& -\left(\delta^{2} / 2\right) \sum\left[\ln \left(x_{i}-\xi\right)-\ln \left(\xi+\lambda-x_{i}\right)\right]^{2}
\end{aligned}
$$

where $\sum$ stands for $\sum_{i=1}^{N}$ and $N$ is the sample size. To do this, we used the function optim of $\mathrm{R}$ language and we set specified initial values of location and scale parameters $\left(\xi_{\text {start }}\right.$ and $\left.\lambda_{\text {start }}\right)$ and the distribution constraints. In 
particular, being JSB a bounded distribution in the interval $[\xi, \xi+\lambda]$ and being the DSDs physically bounded below and above by the minimum and maximum drops diameter, $D_{\min }$ and $D_{\max }$, the initial values of $\xi$ and $\lambda$ are chosen sufficiently below and above the lowest and the highest sample observations, respectively. Thus, $\xi_{\text {start }}$ is set equal to $\left(D_{\min }-\varepsilon_{1}\right)$ and $\lambda_{\text {start }}$ is set equal to $\left(D_{\max }-\xi_{\text {start }}+\varepsilon_{2}\right)$, where $\varepsilon_{1}$ and $\varepsilon_{2}$ are two arbitrarily small quantities. See [1,3] for more details. Alternatively, [2] used a Least Square method applied to theoretical and empirical third order moment to estimate the parameters of JSB. 


\section{Bibliography}

[1] Cugerone, K. and De Michele, C.: Johnson SB as general functional form for raindrop size distribution, Water Resources Research, 51, 6276-6289, doi:10.1002/2014WR016484, 2015.

[2] D'Adderio, L. P., Cugerone, K., Porcù, F., De Michele, C., and Tokay, A.: Capabilities of the Johnson SB distribution in estimating rain variables, Advances in Water Resources, 97, 241-250, doi: 10.1016/j.advwatres.2016.09.017, 2016.

[3] Cugerone, K. and De Michele, C.: Investigating rain drop size distributions in the (L-)skewness-(L-)kurtosis plane, Quarterly Journal of the Royal Meteorological Society, doi:10.1002/qj.3005, 2017.

[4] Johnson NL. 1949. System of frequency curves generated by method of translation. Biometrika, 36, 149-176. 